\title{
Comment \\ Comment on Experimental Determination of the Threshold Dose for Bifidogenic Activity of Dietary 1-Kestose in Rats. Foods 2020, 9, 4
}

\author{
Yihao Shen ${ }^{1}$, Yang Shi ${ }^{1}$ and Zhongke Sun ${ }^{1,2, *(D)}$ \\ 1 Institute of Food and Drug Inspection, Zhoukou Normal University, Zhoukou 466001, China \\ 2 College of Chemistry and Molecular Engineering, Zhengzhou University, Zhengzhou 450001, China \\ * Correspondence: sunzh@daad-alumni.de
}

Received: 4 March 2020; Accepted: 17 April 2020; Published: 21 April 2020

Keywords: prebiotics; bifidogenic activity; Bifidobacterium; fructo-oligosaccharide (FOSs) dose; quantitative real-time polymerase chain reaction (qPCR)

Currently, our group is undertaking a program trying to evaluate the bifidogenic effect/activity of different prebiotics and their dose-effect relationships. Therefore, we were interested to read an article reported the determination of the minimal threshold dose for the bifidogenic activity of dietary 1-kestose in rats published in Foods in December 2019 [1]. It was reported that Bifidobacterium spp. preferentially metabolized fructo-oligosaccharides (FOSs) with a lower degree of polymerization [2]. As the major component of FOSs with the smallest molecular weight, determination of its minimal threshold dose for the bifidogenic activity of 1-kestose in foods is definitely important. However, we have some concerns, especially on the methodological drawbacks, which may be critical to this report.

Watanabe et al. measured cecal microbiota populations using quantitative real-time polymerase chain reaction (qPCR) technology [1]. The 16S rRNA was amplified as a target gene for quantification of Bifidobacterium spp. using a pair of primers (F: GATTCTGGCTCAGGATGAACGC; R: CTGATAGGACGCGACCCCAT). The authors provided a reference (Reference 8 ) for the primers. However, we noticed that paper enumerated Bifidobacterium spp. by traditional cell counting on agar plate other than qPCR amplification [3]. It is obvious the citation is incorrect.

Then, we found the above primers in another paper, which came from the same research group, in which a similar experiment was conducted investigating the effect of 1-kestose within a range of 0.5-5.0\% [4]. In this paper, the primers have the same sequences but different names (Bif LM26F/Bif 228R). Although it was primarily designed based only on the corresponding gene of Bifidobacterium longum subsp. longum $\mathrm{JCM} 1217^{\mathrm{T}}$ (B. longum), this pair of primers was used as the Bifidobacterium genus specific primer. In fact, the primers (Bif LM26F/Bif 228R) were first designed in 2004 [5]. The applicability/university of the primers was evaluated with 10 type strains of Bifidobacterium spp., representing 10 different species. The specificity of the primers was checked with 21 type strains of nonbfidobacerial strains. Thereafter, the primers were used in many studies. However, we now know there are more than 88 species in the genus of Bifidobacterium and the diversity of gut microbiota is quite high [6]. Therefore, we wonder whether the primers are reliable for genus-specific amplification.

To support our speculation, we performed a specificity check of this pair of primers using Primer-BLAST with default settings [7]. Results produced online indicate both the specificity and universality of this pair of primers are questionable. According to this prediction, the primers yield $\sim 230 \mathrm{bp}$ amplicons from different genera of bacteria, targeting the 16S rRNA gene. It was equally efficient for Gardnerella spp., Scardovia inopinata, and Parascardovia denticolens, albeit could amplify many species of Bifidobacterium spp. Unfortunately, representatives of these bacteria were not included in the original study [5]. Efficient detection of many species of Bifidobacterium could only be possible with 
one mismatch in primers. With two mismatches in primers, even Bacillus megaterium, Chryseobacterium indologenes, Synergistales bacterium, and Microbacterium spp. could be amplified as easily as B. animalis. Moreover, many strains belonging to B. saguini, B. callitrichos, B. mongoliense, B. psychraerophilum, B. catenulatum, B. pseudolongum, $B$. reuteri, and B. longum could only be detected with three mismatches in primers. At the same time, more than 12 other genera of bacteria could be positively amplified under such conditions. Details of representative untargeted microbes that could be potentially detected with this pair of primers were summarized (Table 1). Therefore, it seems this pair of primers is neither universal nor specific to Bifidobacterium spp.

Table 1. Primer specificity analysis using Primer-BLAST.

\begin{tabular}{ccc}
\hline Number of Mismatch & Untargeted Microbes & Amplicon Size (bp) \\
\hline \multirow{2}{*}{$\mathbf{1}$} & Gardnerella spp. & 231 \\
& Scardovia inopinata & 235 \\
Parascardovia denticolens & 232 \\
\hline \multirow{2}{*}{$\mathbf{2}$} & Uncultured bacterium & $230-235$ \\
\hline & Uncultured bacterium & $227-235$ \\
& Synergistales bacterium & 229 \\
Microbacterium spp. & 230 \\
& Chryseobacterium indologenes & 160 \\
Bacillus megaterium & 189 \\
\hline \multirow{3}{*}{3} & Slackia spp. & 231 \\
& Raoultibacter spp. & 230 \\
& Microbacterium spp. & $226-232$ \\
& Arabia massiliensis & 230 \\
& Nesterenkonia spp. & 234 \\
& Cellulomonas spp. & $228-231$ \\
& Actinomyces spp. & 238 \\
& Enorma spp. & 227 \\
& Collinsella spp. & 227 \\
Arthrobacter spp. & 232 \\
Lysinimonas spp. & $230-232$ \\
& Eggerthellaceae bacterium & 232 \\
\hline
\end{tabular}

Thirdly, B. pseudolongum was recently identified as almost the sole bifidobacterial species $(>95 \%)$ after FOS (containing 60.6\% 1-kestose) treatment in mice by a comprehensive metagenomic study [8]. Further study indicated that FOS could selectively promote B. pseudolongum proliferation in both the lumen and the mucosa from the cecum to the distal colon in mice [9]. If this is also the case in rats, results obtained with primers having as many as three mismatches, as predicted by Primer-BLAST, may not be reliable.

Fourthly, details of the qPCR analyses were not described in the cited reference by the authors [4], but in another reference [10]. From that paper, we know the authors used $20 \mathrm{ng}$ genomic DNA as a template and SYBR green as fluorescent dye during qPCR, and included melting curves. However, no data were supplied about $\mathrm{Ct}$ values and melting temperatures; these data are important for quality evaluation and reproducibility. From the normalized data demonstrated in Figure 1, it seems the qPCR data were of low quality, as indicated by the large standard error of mean (SEM). Meanwhile, information on repeats was unavailable. 
Lastly, but importantly, the authors concluded that the minimum dose of dietary 1-kestose to induce significant bifidogenic activity in rats was $0.3 \%$ by weight in the diet. In contrast, Tandon et al. reported that intake of FOS appears to result in a statistically significant increase in the abundance of most of the operational taxonomic units (OTUs) belonging to Bifidobacterium in humans, irrespective of amount; even as low as $2.5 \mathrm{~g} / \mathrm{d}$ was administered [11]. In fact, we observed significant stimulation of bifidobacteria using as low as $0.125-0.25 \%$ FOS and other prebiotics during in vitro fermentation, in our unpublished study. Meanwhile, the authors claimed that $0.16 \mathrm{~g} / \mathrm{d} / \mathrm{kg}$ of rat body weight $(0.3 \%$ 1-kestose in feed) was roughly equivalent to $10 \mathrm{~g} / \mathrm{d}(\sim 0.16 \times 60)$ for humans of around $60 \mathrm{~kg}$ body weight. We wonder whether such a calculation is appropriate, as the sensitivity to a reagent in rats and humans is often different. In toxicological studies, the sensitivity index between humans and rats is 6.25 in common [12]. Therefore, we call more attention to the method and conclusion. More studies are required to determine the minimum effective dose of 1-kestose in rats and feeding studies in humans are needed before making a recommendation as a functional food.

Author Contributions: Conceptualization, Y.S. (Yihao Shen) and Z.S.; Writing-original draft, Y.S. (Yihao Shen) and Y.S. (Yang Shi); Writing-review \& editing, Z.S. All authors have read and agreed to the published version of the manuscript.

Funding: This research received no external funding.

Acknowledgments: We thank the authors for replying to our comment and providing many useful references. We express gratitude to reviewers for their constructive suggestions.

Conflicts of Interest: The authors declare no conflict of interest.

\section{References}

1. Watanabe, A.; Kadota, Y.; Yokoyama, H.; Tsuruda, S.; Kamio, R.; Tochio, T.; Shimomura, Y.; Kitaura, Y. Experimental determination of the threshold dose for bifidogenic activity of dietary 1-kestose in rats. Foods 2020, 9, 4. [CrossRef] [PubMed]

2. Ose, R.; Hirano, K.; Maeno, S.; Nakagawa, J.; Salminen, S.; Tochio, T.; Endo, A. The ability of human intestinal anaerobes to metabolize dfferent oligosaccharides: Novel means for microbiota modulation? Anaerobe 2018, 51, 110-119. [CrossRef] [PubMed]

3. Bouhnik, Y.; Vahedi, K.; Achour, L.; Attar, A.; Salfati, J.; Pochart, P.; Marteau, P.; Flourie, B.; Bornet, F.; Rambaud, J.C. Short-chain fructooligosaccharide administration dose-dependently increases fecal bifidobacteria in healthy humans. J. Nutr. 1999, 129, 113-116. [CrossRef] [PubMed]

4. Tochio, T.; Kitaura, Y.; Nakamura, S.; Sugawa, C.; Takahashi, M.; Endo, A.; Shimomura, Y. An Alteration in the cecal microbiota composition by feeding of 1-kestose results in a marked increase in the cecal butyrate content in rats. PLoS ONE 2016, 11, e0166850. [CrossRef] [PubMed]

5. Gueimonde, M.; Tölkkö, S.; Korpimäki, T.; Salminen, S. New real-time quantitative PCR procedure for quantification of bifidobacteria in human fecal samples. Appl. Environ. Microbiol. 2004, 70, 4165-4169. [CrossRef] [PubMed]

6. LPSN, List of Prokaryotic names with Standing in Nomenclature. Available online: https://lpsn.dsmz.de/ search? word=bifidobacterium (accessed on 1 March 2020).

7. Ye, J.; Coulouris, G.; Zaretskaya, I.; Cutcutache, I.; Rozen, S.; Madden, T. Primer-BLAST: A tool to design target-specific primers for polymerase chain reaction. BMC Bioinform. 2012, 13, 134. [CrossRef] [PubMed]

8. Takahashi, S.; Tomita, J.; Nishioka, K.; Hisada, T.; Nishijima, M. Development of a prokaryotic universal primer for simultaneous analysis of Bacteria and Archaea using next-generation sequencing. PLoS ONE 2014, 9, e105592. [CrossRef] [PubMed]

9. Mao, B.; Gu, J.; Li, D.; Cui, S.; Zhao, J.; Zhang, H.; Chen, W. Effects of different doses of fructooligosaccharides (FOS) on the composition of mice fecal microbiota, especially the Bifidobacterium composition. Nutrients 2018, 10, 1105. [CrossRef] [PubMed]

10. Gu, J.; Mao, B.; Cui, S.; Liu, X.; Zhang, H.; Zhao, J.; Chen, W. Metagenomic insights into the effects of fructooligosaccharides (FOS) on the composition of luminal and mucosal microbiota in C57BL/6J mice, especially the Bifidobacterium composition. Nutrients 2019, 11, 2431. [CrossRef] [PubMed] 
11. Tandon, D.; Haque, M.M.; Gote, M.; Jain, M.; Bhaduri, A.; Dubey, A.K.; Mande, S.S. A prospective randomized, double-blind, placebo-controlled, dose-response relationship study to investigate efficacy of fructo-oligosaccharides (FOS) on human gut microflora. Sci. Rep. 2019, 9, 5473. [CrossRef] [PubMed]

12. Yue, Z.; Chen, Y.; Song, Y.; Zhang, J.; Yang, X.; Wang, J.; Li, L.; Sun, Z. Effect of acrylamide on glucose homeostasis in female rats and its mechanisms. Food Chem. Toxicol. 2020, 135, 110894. [CrossRef] [PubMed]

(C) 2020 by the authors. Licensee MDPI, Basel, Switzerland. This article is an open access article distributed under the terms and conditions of the Creative Commons Attribution (CC BY) license (http://creativecommons.org/licenses/by/4.0/). 